\title{
Catalogue of the type material of Phasmatodea (Insecta) deposited in Brazilian Museums
}

\author{
Oliver Zompro ${ }^{1} \&$ Fernando C. de Domenico ${ }^{2}$ \\ 1. Max-Planck-Institut für Limnologie, AG Tropenökologie, August-Thienemann-Straße 2, 24306 Plön, Germany. \\ (o.zompro@sungaya.de) \\ 2. Museu da Zoologia da Universidade São Paulo, Av. Nazaré, 481, Ipiranga, 04263-000 São Paulo, SP, Brazil. (fcdomenico@usp.br)
}

\begin{abstract}
The type material of Phasmatodea deposited in Brazilian museums and institutions is listed for the first time. New synonyms are proposed: Phibalosoma paulense Toledo Piza, 1938, Phibalosoma rochai Toledo Piza, 1938, Bacteria tuberculata Toledo Piza, 1938 and Bacteria tuberculata var. argentina Toledo Piza, 1938 are junior synonyms of Cladomorphus phyllinus (Gray, 1835). Nineteen new combinations are established.
\end{abstract}

KEYWORDS. Phasmatodea, type material, Brazil, new synonyms, new combinations.

RESUMO. Catálogo do material-tipo de Phasmatodea (Insecta) depositado em museus brasileiros. O material-tipo de Phasmatodea depositado em museus e instituições brasileiras é listado pela primeira vez. Novas sinonímias são propostas: Phibalosoma paulense Toledo Piza, 1938, Phibalosoma rochai Toledo Piza, 1938, Bacteria tuberculata Toledo Piza, 1938 e Bacteria tuberculata var. argentina Toledo Piza, 1938 são sinônimos júnior de Cladomorphus phyllinus (Gray, 1835). Dezenove novas combinações são estabelecidas.

PALAVRAS-CHAVE. Phasmatodea, material-tipo, Brasil, novas sinonímias, novas combinações.

More than 200 species of Phasmatodea are described from Brazil. The type material of Phasmatodea deposited in Brazilian museums mainly belongs to the 38 species described by Prof. Dr. Salvador de Toledo Piza Jr. (1898-1988), even though not all of these species were collected in Brazil. Further species have been described by ZoMPRo \& Adis (2001) and ZoMPro (2004, 2005) .

The type material is housed in the following collections: Toledo Piza Collection (ESLQTP), Departamento de Entomologia, Fitopatologia e Zoologia Agrícola, Escola Superior de Agricultura "Luiz de Queiroz", Piracicaba, São Paulo - this collection, arranged in six wooden drawers with glass top (nos. 24/58, 25/59, $26 / 60,27 / 61,28 / 57$ and 61/62), contains the majority of the species described by Toledo Piza; Museu de Zoologia da Universidade São Paulo (MZSP), São Paulo - this collection contains about one dozen drawers of pinned material and several jars with material in alcohol, with types of Toledo Piza and Zompro \& Adis; Museu Paraense Emílio Goeldi (MPEG), Belém, Pará; Instituto Biológico de São Paulo (IBSP), São Paulo.

All type specimens have been examined in the collections concerned. The type status of all specimens has been confirmed by a comparison with the original descriptions. The taxa are listed in alphabetical order of the species name in the list below. The systematic attachment of the species in this catalogue follows BRADLEY \& Galil (1977) and includes the changes published by ZoMPro $(2001,2004,2005)$. The following format is used:

species AutHOR, reference [original genus]

[Data as mentioned in the original description: locality; date; collector; additional data]
COLLECTION: data of labels of type specimens [valid name]

Observations

List of type material

affinis Toledo Piza, 1936b:281 [Canuleius]

[Type, o: Alto da Serra (S. Paulo); no date mentioned; Schwebel leg.; Nr. 15294.]

MZSP: holotype, $:$ : 15294, S. Paulo; 93.059.

[Canuleius affinis Toledo Piza, 1936] Heteronemiidae: Canuleiini.

"Alto da Serra" probably refers to the present Paranapiacaba city, SP, Brazil.

albogranulatus Toledo PizA, 1938b:9 [Ceroys]

[Type, ơ: São Paulo, Alto da Serra; no date mentioned; Schwalb leg.]

ESLQTP: holotype, ơ: São Paulo, Alto da Serra, coll. E. Schw., 30-6-32.

[Ceroys (Ceroys) albogranulatus Toledo Piza, 1938] Heteronemiidae: Canuleiini.

"Alto da Serra" probably refers to the present Paranapiacaba city, SP, Brazil.

auritus Toledo Piza, 1936b:287, pl. 2: 2 [Ceroys]

[Type, :: S. Paulo; no date mentioned; A. Baer leg.]

MZSP: holotype,,$: 14.882 ; 92.950$.

[Ceroys toledopizai Zompro, 2003] Heteronemiidae: Canuleiini.

Replacement name, preoccupied by Ceroys auritus (Burmeister, 1838).

bahiensis Toledo Piza, 1938a:42, fig. 3 [Pseudobacteria] 
[\%: Bahia; no date mentioned; Camargo col.]

ESLQTP: holotype, : Bahia, Jequié, Camargo leg., XII.32. [Phanocloidea bahiensis (Toledo Piza, 1938) comb. nov.]

"Anareolatae": Diapheromeridae: Diapheromerinae: Diapheromerini.

bispinosus Toledo Piza, 1936b:282, fig. 1 [Canuleius] [Type, o: Bahia; 1932; Camargo leg.]

MZSP: holotype, ơ: XII.32, Bahia, Jequié, Camargo leg.; 93.061.

[Canuleius bispinosus Toledo Piza, 1936]

Heteronemiidae: Canuleiini.

bispinosus Toledo Piza, 1939b:114 [Cladoxerus]

[Type, : Orán (Salta), Argentina; no date mentioned; J.

B. Daguerre leg.; $\mathrm{n}^{\circ}$. 34786.]

ESLQTP: not traced.

[Cladoxerus bispinosus Toledo Piza, 1939] "Anareolatae": Diapheromeridae: Cladomorphinae.

boliviana Toledo PizA, 1939b:115 [Heteronemia]

[Type, o: Bolivia; no date mentioned; J. Steinbach leg.; no. 12138.]

ESLQTP: not traced.

[Dyme boliviana (Toledo Piza, 1939) comb. nov.]

"Anareolatae": Diapheromeridae: Diapheromerinae:

Diapheromerini.

This species can tentatively be placed in Dyme Stål, 1875, but the diagnosis does not allow a definite assignment.

bosqi Toledo Piza, 1938b:4 [Phthoa]

[Types, of, o: Misiones (Republica Argentina); no date mentioned; Juan M. Bosq leg.]

ESLQTP: syntypes, $\sigma^{\top}$, : Juan M. Bosq, Misiones (Arg.). [Paraleptynia bosqi (Toledo Piza, 1938) comb. nov.] Heteronemiidae: Paraleptyniini.

brasiliensis Toledo PIZA, 1938b:4 [Phthoa]

[Type, o: Murtinho (E. de Matto Grosso); no date mentioned; R. Spitz col.]

ESLQTP: holotype,, : 22.030.

[Globocalynda brasiliensis (Toledo Piza, 1938) comb.

nov.] "Anareolatae": Diapheromeridae: Diapheromerinae:

Diapheromerini.

The head is too long for a Globocalynda Zompro, 2001, possibly a new genus is required. The locality "E. de Matto Grosso" mentioned in the original description refers to the present Rondônia state, Brazil.

brasiliensis Toledo PizA, 1938b:8, fig. 8 [Dixippus] [Type, o: Brasil (?); no date mentioned; Luederwaldt col.] ESLQTP: holotype, o: 14.873.

[Hermagoras hosei (Kirby, 1896)] "Anareolatae": Diapheromeridae: Lonchodinae: Lonchodini.

Synonymized by BRAGG, 2001: 461. Definitely from Asia.

brevipes Toledo Piza, 1936b:284 [Canuleius]

[Type, : : São Paulo (Alto da Serra); no date mentioned; D. Braz col.]

IBSP: holotype, o: São Paulo, Alto da Serra, IV-1936, Coll.

Donias Braz, 1121.
[Canuleius brevipes Toledo Piza, 1936] Heteronemiidae: Canuleiini.

"Alto da Serra" probably refers to the present city of Paranapiacaba, SP, Brazil.

brunneri Toledo Piza, 1936b:288, pl. 2: 5 [Ceroys]

[Type, o: Rio de Janeiro, Macahé; no date mentioned; Garbe leg.]

MZSP: holotype,

[Ceroys brunneri Toledo Piza, 1936] Heteronemiidae: Canuleiini.

cearensis Toledo Piza, 1938b:6, fig. 7 [Wattenwylia] [Types, 2 : Ceará; no date mentioned; Prof. Dias da Rocha leg.]

ESLQTP: not traced.

[Wattenwylia cearensis Toledo Piza, 1938] "Anareolatae": Diapheromeridae: Cladomorphinae.

chacoensis Toledo Piza, 1939b:113 [Bacteria]

[ơ. Argentina: Chaco; no date mentioned; F. Ohnheiser leg.; no. 34194.]

ESLQTP: not traced.

[Bacteria chacoensis Toledo Piza, 1939] "Anareolatae":

Diapheromerinae: Diapheromerini.

The description does not allow a generic assignment, but it does not definitely belong to Bacteria St. Fargeau \& Audinet-Serville, 1827.

corallinus Toledo Piza, 1936b:282 pl. 2: 3 [Canuleius] [Types, $20^{\text {: }}$ : Alto da Serra (S. Paulo); no date mentioned; Schwebel leg.]

MZSP: syntype, ơ: 14.893; 93.056; syntype, ơ: 15585, São

Paulo Est. Alto Serra.

[Canuleius corallinus Toledo Piza, 1936] Heteronemiidae: Canuleiini.

"Alto da Serra" probably refers to the present city of Paranapiacaba, SP, Brazil.

crassithorax Toledo PizA, 1937:2 [Olcyphides]

[Type, : Santa Catharina (Colonia Hansa); 1907; Ehrhardt leg.]

ESLQTP: holotype, $:$ : St. Catharina, col. Hansa, anno 1907. [Metriophasma (Metriophasma) crassithorax (Toledo Piza, 1937) comb. nov.] Pseudophasmatidae: Stratocleinae. "Colonia Hansa" probably refers to a village at the city of Joinville, SC, Brazil (NeIva, 1930).

cryphaleus var. dentatus Toledo PizA, 1938a:41 [Cladoxerus]

[o: S. Paulo (Capital); no date mentioned; no collector mentioned.]

ESLQTP: holotype, o: São Paulo, Capital, X-22.

[Baculum dentatum (Toledo Piza, 1938) comb. nov.]

"Anareolatae": Diapheromeridae: Cladomorphinae.

debilis Toledo Piza, 1938b:2, fig. 1 [Gratidia]

[Type, o: Ceará; no date mentioned; Prof. Dias da Rocha leg.]

ESLQTP: not traced.

[Xiphophasma debilis (Toledo Piza, 1938) comb. nov.] 
Heteronemiidae: Paraleptyniini.

dentatus Toledo Piza, 1937:7 [Stratocles]

[ơ: Matto Grosso (Três Lagoas); Novembro de 1929; Coll. R. Spitz.]

ESLQTP: holotype, ơ: Matto Grosso, Três Lagoas, Coll. R. Spitz, XI.29.

[Paraphasma dentatum (Toledo Piza, 1937) comb. nov.] Pseudophasmatidae: Stratocleinae.

The locality "Matto Grosso" mentioned in the original description and on the label refers to the present Mato Grosso do Sul state, Brazil.

dubius Toledo Piza, 1937:3 [Olcyphides]

[Type, o: São Paulo; no date mentioned; Schwebel col.; no. 14.896.]

ESALQTP: syntype, ơ: São Paulo Capital coll. E. Schw. II.96; syntype, ơ: 14.896.

[Perliodes dubius (Toledo Piza, 1937) comb. nov.] Pseudophasmatidae: Xerosomatinae: Prexaspini.

Toledo Piza (1937) refers to the label data of both specimens. In the description he states "Typo no Museu Paulista". The use of the singular must be considered as erroneous.

evoneobertii Zompro \& AdIs, 2001:294, fig. 1 [Echetlus] [Holotype, ơ: Brazil, Boa Esperança do Sul; 18.II.1998; Cerignoni, J. A. Paratypes, 2 , 10 eggs: Brazil, PiracicabaESLQ/USP; IX.2000; Bernardi, E. B; criado em laboratório em folhas de Eucalyptus sp.]

MZSP: holotype, o: Brazil, Boa Esperança do Sul, Cerignoni, J. A., 18.II.1998; Paratypes: 2 \&, 10 eggs: Brazil, Piracicaba-Esalq/USP, Bernardi, E.B., IX.2000, criato em laboratorio em folias (sic) Eucalyptus sp.

[Echetlus evoneobertii Zompro \& Adis, 2001] "Anareolatae": Phasmatidae: Platycraninae.

Further type material in coll. O. Zompro and Australian National Collection, Canberra, Australia.

fischeri TolEdo PizA, 1936b:283 [Canuleius]

[Type, o: Rio de Janeiro; no date mentioned; J. Vic. Azevedo leg.]

MZSP: holotype, o: Rio de Janeiro ?.

[Canuleius fischeri Toledo Piza, 1936] Heteronemiidae: Canuleiini.

flagelloantennata ZoMPro, 2005:137, fig. 3 [Otocraniella] [Holotype, o: [Brazil] Chapéu de Sol - Serra do Cipó MG; 7.XII.1969; Montouchet col.; Papered.]

MZSP: holotype, : Chapéu de Sol - Serra do Cipó - MG, 7.XII.1969, Montouchet col.

[Otocraniella flagelloantennata Zompro, 2004]

"Anareolatae": Diapheromeridae: Cladomorphinae.

foliata Toledo PizA, 1938b:6, fig. 4-6 [Wattenwylia] [Types, 4 : Ceará; no date mentioned; Prof. Dias da Rocha leg.]

ESLQTP: not traced.

[Wattenwylia foliata Toledo Piza, 1938] "Anareolatae": Diapheromeridae: Cladomorphinae.

According to the original description, two female types should be housed in the author's Collection and another two at "Museu Rocha, Fortaleza, Ceará, Brazil". However, "Museu Rocha" does not exist anylonger and its collection would have been transferred to the Centro de Ciências Agrárias (CCAC), Universidade Federal do Ceará, Fortaleza, Ceará, Brazil. The CCAC collection has not been visited, but provided a list of material. This did not include phasmatodeans.

\section{fragilis Toledo Piza, 1938b:2 [Gratidia]}

[Type, ơ: Ceará; no date mentioned; Prof. Dias da Rocha leg.]

ESLQTP: not traced.

[Xiphophasma fragilis (Toledo Piza, 1938) comb. nov.] Heteronemiidae: Paraleptyniini.

fulgens ZомРRо, 2005:138, fig. 4 [Echetlus]

[Holotype, : Brasil, MT, Barra dos Bugres, R. E. Serra das Araras; 23.I.1986; no collector mentioned.]

MPEG: holotype, $:$ : Brasil, MT, Barra dos Bugres, R. E. Serra das Araras, 23.I.1986.

[Echetlus fulgens Zompro, 2004] "Anareolatae": Phasmatidae: Platycraninae.

grandis Toledo PizA, 1936b:280, pl. 2: 1 [Canuleius]

[Type, o: Alto da Serra (S. Paulo), Brasil; no date mentioned; Schwebel leg.; nr. 14.800. Type, : Castro (Paraná), Brasil; no date mentioned; Garbe leg.; nr. 14.893b.]

MZSP: syntype,,$: 14800$, S. Paulo; 93.057; Syntype, 14893b, Paraná; 93.058.

[Canuleius grandis Toledo Piza, 1936] Heteronemiidae: Canuleiini.

"Alto da Serra" probably refers to the present Paranapiacaba city, SP, Brazil.

heymonsi Toledo Piza, 1936b:291, pl. 2: 6 [Miroceroys] [Type, o, Paratypes, 2 o: São Paulo (Alto da Serra); no date mentioned; D. Braz col. Do Instituto Biologico de São Paulo.]

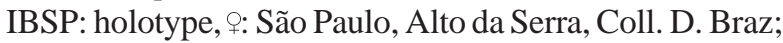
Paratype, 1 + nymph: São Paulo, Alto da Serra, coll. Braz, 1119.

[Ceroys (Miroceroys) heymonsi (Toledo Piza, 1936) comb. nov.] Heteronemiidae: Canuleiini.

In the original description, the author mentions two juvenile females (paratypes). Only one could be traced. "Alto da Serra" probably refers to the present Paranapiacaba city, SP, Brazil.

imbe Toledo Piza, 1939a:444, fig. 1 [Otocrania]

[Type, : Água Preta, E. da Bahia, Brasil; 1938; Agr. Pedrito Silva col.]

ESLQTP: holotype, §: Col. Em Imbé, 10-12-938, Agua Preta, E. da Bahia, P. Silva.

[Otocrania imbe Toledo Piza, 1939] "Anareolatae": Diapheromeridae: Cladomorphinae.

libidinosus Toledo Piza, 1943:290, fig. 3-4 [Acanthoderus]

[Types, ơ, ๑: Mogí das Cruzes, E. de S. Paulo; XI-1939; M. 
Carrera col.; collected in copula]

ESLQTP: syntypes, ơ,, , mounted on one pin: São Paulo, Mogy da Cruzes (in cop.), XI-939, M. Carrera coll. [Canuleius libidinosus (Toledo Piza, 1943) comb. nov.] Heteronemiidae: Canuleiini.

longipes Toledo PIZA, 1938a:42, fig. 1 [Pseudobacteria] [Type, ơ: Rio de Janeiro; no date mentioned; no collector mentioned; No. 16.047.]

ESLQTP: holotype, ơ: 16.047.

[Paracalynda longipes (Toledo Piza, 1938) comb. nov.]

"Anareolatae": Diapheromeridae: Diapheromerinae: Diapheromerini.

The species seems to belong to a yet undescribed genus closely related to Paracalynda Zompro, 2001.

melzeri Toledo Piza, 1937:6 [Planudes]

[o: S. Paulo (Bosque da Saúde); no date mentioned; Julius Melzer leg.]

ESLQTP: holotype, Melzer leg.

[Planudes melzeri Toledo Piza, 1937] Pseudophasmatidae: Xerosomatinae: Prexaspini.

"Bosque da Saúde" refers to a district in São Paulo city, SP, Brazil.

modestus Toledo Piza, 1985b:101 [Stratocles]

[ơ. Serra do Navio, Amapá; 10-XI-57; Lenko col.]

ESLQTP: holotype, ơ: S. do Navio, 10-X-57; Amapá; Lenko.

[Citrina modesta (Toledo Piza, 1985) comb. nov.] Pseudophasmatidae: Stratocleinae.

multispinosus Toledo Piza, 1936b:287 [Ceroys]

[Type, ९: S. Paulo, Alto da Serra; no date mentioned; Schwebel leg.]

MZSP: holotype, $: 14.893$ a.; 92.947.

[Ceroys (Ceroys) spinosus Zompro, 2004] Heteronemiidae: Canuleiini.

Replacement name, preoccupied by Ceroys multispinosus Audinet-Serville, 1838. "Alto da Serra" probably refers to the present Paranapiacaba city, SP, Brazil.

nigrovittata Toledo PizA, 1939a:446, fig. 2 [Donusa]

[Type, ơ: Salto Grande, E. de S. Paulo, Brasil; no date mentioned; Lüderwaldt col.]

ESLQTP: holotype, ơ: without label.

[Neophasma nigrovittata (Toledo Piza, 1939) comb. nov.] Pseudophasmatidae: Pseudophasmatinae: Anisomorphini.

ocellatus Toledo Piza, 1937:2 [Olcyphides]

[Type, o: Santa Catharina (Blumenau); 1929; no collector mentioned.]

ESLQTP: holotype, ơ: St. Cattharina, Blumenau, Nat. Schaefer ott. 1929.

[Metriophasma (Metriophasma) ocellatum (Toledo Piza, 1937) comb. nov.] Pseudophasmatidae: Stratocleinae.

paradiacanthoides Toledo Piza, 1938b:8, fig. 3 [Dilophocephalus]

[Type, ơ: Brasil (?); no date mentioned; no collector mentioned; $\mathrm{n}^{\circ} 14873$.]

ESLQTP: holotype, ơ: 14.873.

[Paradiacantha acanthocephala (de Haan, 1842)]

"Anareolatae": Necrosciinae.

Synonymized by BRAGG, 2001: 591. Definitely from Asia.

paulense Toledo PizA, 1938a:41, fig. 2 [Phibalosoma]

[ơ: São Paulo; no date mentioned; no collector mentioned.]

ESLQTP: holotype, ơ: São Paulo.

[Cladomorphus phyllinus (Gray, 1835) syn. nov.]

"Anareolatae": Diapheromeridae: Cladomorphinae.

platensis Toledo PizA, 1939b:115 [Leptinia]

[ $\checkmark$. Argentina; no date mentioned; Eng. Agr. C. A. Lizer y

Trelles leg.]

ESLQTP: not traced.

[Paraleptynia platensis (Toledo Piza, 1939) comb. nov.] Heteronemiidae: Paraleptyniini.

redtenbacheri TolEDo PIZA, 1936b:290 [Miroceroys]

[Type, ơ: Rio de Janeiro, Serra de Macahé; no date mentioned; Garbe leg.]

MZSP: holotype, ơ: Serra de Macahé ; 92.952.

[Ceroys (Miroceroys) redtenbacheri (Toledo Piza, 1936)

comb. nov.] Heteronemiidae: Canuleiini.

rochai Toledo Piza, 1938b:1, fig. 2 [Phibalosoma]

[Type, o: Ceará; no date mentioned; no collector mentioned.]

ESLQTP: not traced.

[Cladomorphus phyllinus (Gray, 1835) syn. nov.]

"Anareolatae": Diapheromeridae: Cladomorphinae.

scaber Toledo PizA, 1936b:286, pl. 2: 1 [Ceroys]

[?: S. Paulo, Alto da Serra; no date mentioned; Schwebel leg.]

MZSP: holotype,

[Ceroys scaber Toledo Piza, 1936] Heteronemiidae: Canuleiini.

"Alto da Serra" probably refers to the present Paranapiacaba city, SP, Brazil.

spatulatus Toledo Piza, 1937:5 [Damasippus]

[ơ: Serra de Macahé, E. do Rio do Janeiro; no date mentioned; no collector mentioned.]

MZSP: holotype, ơ: Serra de Macahé, E. d. Rio; 92.956.

[Damasippus spatulatus Toledo Piza, 1937] Prisopodidae:

Prisopodinae: Prisopodini.

tuberculata Toledo PizA, 1936a:98, fig. 1 [Bacteria]

[Type, o: São Paulo, Brasília; no date mentioned; Agrn.

Victor Valentie de Oliveria col.]

ESLQTP: holotype, : no data.

[Cladomorphus phyllinus (Gray, 1835) syn. nov.]

"Anareolatae": Diapheromeridae: Cladomorphinae.

"Brasília" probably refers to a railroad station at Piratininga city, SP, Brazil (NeIVA, 1930).

tuberculata var. argentina Toledo PIZA, 1939b:113 [Bacteria]

[९. Jujuy, Dto. C. Ovejero, Santa Clara, Argentina; no date 
mentioned; Dr. Aristides Fiora col.; no. 36991.]

ESLQTP: not traced.

[Cladomorphus phyllinus (Gray, 1835) syn. nov.]

"Anareolatae": Diapheromeridae: Cladomorphinae.

vetus Toledo Piza, 1936b:281 [Canuleius]

[Type, o: Sta. Catharina; 1901; Ehrhardt leg.]

MZSP: holotype, o: S. Cath. Herhardt.

[Canuleius vetus Toledo-Piza, 1936] Heteronemiidae:

Canuleiini.

vittata Toledo PizA, 1985a:1 [Oestrophora]

[Type, ơ: Boracéia, Salesópolis, SP; 20.11.63; Rabello col. Paratype, o: Mury, Nova Friburgo, R. J.; 12/76; Cred \& Guimaraes col.]

ESLQTP: holotype, ơ: Est. Biol. Boracéia, Salesópolis, S. P., 850m., Rabello col., 20.XI.63; 10; Hoya. Paratype, ơ: Mury, Nova Friburgo, Rio de Janeiro - Br. XII.1976, Cred \& Guimarães col.

[Prexaspes (Elasia) vittata (Toledo Piza, 1985) comb. nov.] Pseudophasmatidae: Xerosomatinae: Prexaspini.

Acknowledgments. The authors wish to thank Prof. Dr. Wolfgang Junk and Prof. Dr. Joachim Adis, who financed the first author's trip to Brazil by a grant from the Tropical Ecology Working Group of the Max-Planck-Institute for Limnology, Plön, Germany. Dr. Célio Magalhães, Dr. José Albertino Rafael, Dr. Augusto L. Henriques, Dr. José Wellington de Moraes and Dr. Suzana Ketelhut supported during the author's stay in Manaus; Dra. Ana Lucia Nunes Guthjahr and Dr. Ekkehardt Guthjahr provided hospitality and support in Belém; Dra. Eliana Marques Cancello and the family Llhano supported in São Paulo and Prof. Dr. Evoneo Berti Filho provided hospitality and support in Piracicaba.

\section{REFERENCES}

Bragg, P. E. 2001. Phasmids of Borneo. Kota Kinabalu, Sabah, Natural History Publikations. 771p.
Bradley, J. C. \& Galil, B. S. 1977. The taxonomic arrangement of the Phasmatodea with keys to the subfamilies and tribes. Proceedings of the Entomological Society of Washington 79(2):176-208.

Neiva, S. H. L. 1930. Guia Postal (Geográphico) da República dos Estados Unidos do Brasil. Rio de Janeiro, Typographia da Directoria Geral dos Correios. v.1, 928p.

Toledo Piza, S. DE. 1936a. Um novo Phasmida do Brasil (Orth.). Revista de Entomologia 6(1):98-100.

_. 1936b. Os Phasmidas do Museu Paulista. I. Phasmidae, Bacillinae. Revista de Entomologia 6(2):280-292, pl. 2.

__. 1937. Os Phasmidas do Museu Paulista. II. Phasmidae, Phasminae. Revista de Entomologia 7(1):1-8.

_. 1938a. Os Phasmidas do Museu Paulista. III. Phasmidae, Cladoxerinae. Revista de Entomologia 8(1-2):40-44.

_. 1938b. Novos Phasmidas do Brasil e da Argentina (Orth.). Revista de Entomologia 9(1-2):1-11.

. 1939a. Dois novos Phasmidas do Brasil (Orth. ). Revista de Entomologia 10(2):444-446.

. 1939b. Phasmidas do Museu Argentino de Sciencias Naturaes. Physis 17:113-116.

_.. 1943. Um Proscopiida e um Phasmida novos do Brasil (Orth.). Revista de Entomologia 14(1-2):287-291.

- 1985a. A segunda espécie do gen. Oestrophora (Phasmatodea: Phylliidae). Revista de Agricultura 60(1):1-2.

1985b. Um novo Stratocles do Brasil (Phasmatodea: Phylliidae). Revista de Agricultura 60(2):101-102.

Zompro, O. 2001. A generic revision of the insect order Phasmatodea: the New World genera of the stick insect subfamily Diapheromeridae: Diapheromerinae = Heteronemiidae: Heteronemiinae sensu Bradley \& Galil, 1977. Revue Suisse de Zoologie 108(1):189-255.

—. 2004. Revision of the genera of the Areolatae, including the status of Timema and Agathemera (Insecta: Phasmatodea). Abhandlungen des Naturwissenschaftlichen Vereins in Hamburg (NF) 371:1-327.

-_. 2005. A key to the stick-insect genera of the "Anareolatae" of the New World (Insecta: Phasmatodea). Studies on Neotropical Fauna and Environment 39(2): $133-144$

Zompro, O. \& Adis, J. 2001. A new species of Phasmatodea of the genus Echetlus Stål. Revista de Agricultura 76(2):291-297. 